\title{
Exponential Stability of Linear Discrete Systems with Multiple Delays
}

\author{
J. Baštinec, ${ }^{1}$ H. Demchenko, ${ }^{2}$ J. Diblík ${ }^{D},{ }^{1}$ and D. Ya. Khusainov ${ }^{1}$ \\ ${ }^{1}$ Brno University of Technology, CEITEC-Central European Institute of Technology, Brno, Czech Republic \\ ${ }^{2}$ Brno University of Technology, Faculty of Electrical Engineering and Communication Brno, Czech Republic \\ Correspondence should be addressed to J. Diblík; diblik@feec.vutbr.cz
}

Received 14 January 2018; Accepted 8 April 2018; Published 1 August 2018

Academic Editor: Pasquale Candito

Copyright (C) 2018 J. Baštinec et al. This is an open access article distributed under the Creative Commons Attribution License, which permits unrestricted use, distribution, and reproduction in any medium, provided the original work is properly cited.

\begin{abstract}
The paper investigates the exponential stability and exponential estimate of the norms of solutions to a linear system of difference equations with multiple delays $x(k+1)=A x(k)+\sum_{i=1}^{s} B_{i} x\left(k-m_{i}\right), k=0,1, \ldots$, where $s \in \mathbb{N}, A$ and $B_{i}$ are square matrices, and $m_{i} \in \mathbb{N}$. New criterion for exponential stability is proved by the Lyapunov method. An estimate of the norm of solutions is given as well and relations to the well-known results are discussed.
\end{abstract}

\section{Preliminaries}

The investigation of the stability of linear difference systems with delay is a constant priority of research. We refer, for example, to [1-14] and to the references therein.

The paper considers the exponential stability of linear discrete systems with multiple delays

$$
x(k+1)=A x(k)+\sum_{i=1}^{s} B_{i} x\left(k-m_{i}\right), \quad k=0,1, \ldots
$$

where $s \in \mathbb{N}, A$ and $B_{i}$ are $n \times n$ matrices, and $m_{i} \in \mathbb{N}$. For (1) exponential-type stability and exponential estimate of the rate of convergence of solutions are derived.

Set $m:=\max \left\{m_{1}, \ldots, m_{s}\right\}$. The initial Cauchy problem for system (1) is as follows:

$$
x(k)=x_{k} \in \mathbb{R}, \quad k=-m,-m+1, \ldots
$$

For a vector $x=\left(x_{1}, \ldots, x_{n}\right)^{T}$, we define $|x|^{2}:=\sum_{i=1}^{n} x_{i}^{2}$. Let $\rho(A)$ be the spectral radius of the matrix $A$. Denote by $\lambda_{\max }(\mathscr{A})$ and $\lambda_{\text {min }}(\mathscr{A})$ the maximum and the minimum eigenvalues, respectively, of a symmetric matrix $\mathscr{A}$ and define $\varphi(\mathscr{A}):=\lambda_{\max }(\mathscr{A}) \lambda_{\min }^{-1}(\mathscr{A})$. For a given matrix $\mathscr{B}$, we use the norm defined by $|\mathscr{B}|^{2}:=\lambda_{\text {max }}\left(\mathscr{B}^{T} \mathscr{B}\right)$. In the paper, assume $|A|+\sum_{i=1}^{s}\left|B_{i}\right|>0$.
The trivial solution $x(k)=0, k=-m,-m+1, \ldots$ of $(1)$ is called Lyapunov exponentially stable if there exist constants $N>0$ and $\theta \in(0,1)$ such that, for an arbitrary solution $x=$ $x(k)$ of (1),

$$
|x(k)| \leq N\|x(0)\|_{m} \theta^{k}, \quad k=1,2, \ldots
$$

where

$$
\|x(0)\|_{m}:=\max \{|x(i)|, i=-m,-m+1, \ldots, 0\} .
$$

For the foundations of stability theory to difference equations, we refer, e.g., to $[15,16]$.

As it is customary, the asymptotic stability of (1) can be investigated by analyzing the roots of the related characteristic equation. The characteristic equation relevant to (1) is a polynomial equation of degree $(m+1) n$. For large $m$ and $n$, it is impossible, in a general case, to solve such a problem. For example, the Schur-Cohn criterion $[16,17]$ is not applied because the computer calculation is too time-consuming.

Below, the exponential stability of (1) is analyzed by the second Lyapunov method and the following well-known result is utilized: if $\rho(A)<1$, then the Lyapunov matrix equation

$$
A^{T} H A-H=-C
$$

has a unique solution, a positive definite symmetric matrix $H$ for an arbitrary positive definite symmetric $n \times n$ matrix $C$ (we refer, for example, to [16]). 
In Section 2, the exponential stability of system (1) and exponential estimates of solutions are investigated. Concluding remarks and relations to the well-known results are included in Section 3.

\section{Exponential Stability}

Let $\gamma>1$ be a parameter. Define auxiliary numbers

$$
\begin{aligned}
L_{1} & :=\gamma\left[\lambda_{\max }(H)-\lambda_{\min }(C)+\sum_{i=1}^{s}\left|A^{T} H B_{i}\right|\right], \\
L_{2} & :=\lambda_{\min }(H)-\frac{1}{2} \gamma \varphi(H)\left[2 \sum_{i=1}^{s} \gamma^{m_{i}}\left|A^{T} H B_{i}\right|\right. \\
& \left.+\sum_{i, j=1}^{s}\left[\gamma^{m_{i}+1}+\gamma^{m_{j}+1}\right]\left|B_{i}^{T} H B_{j}\right|\right], \\
L_{3} & :=\lambda_{\min }(C)-\sum_{i=1}^{s}\left|A^{T} H B_{i}\right|-\frac{\gamma-1}{\gamma} \lambda_{\max }(H)-\frac{1}{2} \\
& \cdot \varphi^{2}(H)\left[2 \sum_{i=1}^{s} \gamma^{m_{i}}\left|A^{T} H B_{i}\right|\right. \\
& \left.+\sum_{i, j=1}^{s}\left[\gamma^{m_{i}+1}+\gamma^{m_{j}+1}\right]\left|B_{i}^{T} H B_{j}\right|\right] .
\end{aligned}
$$

Theorem 1. Let $\rho(A)<1, C$ be a fixed positive definite symmetric $n \times n$ matrix, let matrix $H$ solve the equation (5), and, for a fixed $\gamma>1$, let $L_{1}>0, L_{2}>0, L_{3} \geq 0$. Then, system (1) is exponentially stable and, for an arbitrary solution $x=x(k)$, the estimate

$$
|x(k)| \leq \sqrt{\varphi(H)}\|x(0)\|_{m} \gamma^{-k / 2}, \quad k \geq 1
$$

holds.

Proof. For the Lyapunov function $V(x, k):=\gamma^{k} x^{T} H x$, inequalities

$$
\gamma^{k} \lambda_{\min }(H)|x|^{2} \leq V(x, k) \leq \gamma^{k} \lambda_{\max }(H)|x|^{2}
$$

hold. Let $\delta:=\varepsilon / \sqrt{\varphi(H)}$ where $\varepsilon>0$ is given. Let a solution $x(k)$ of (1) satisfy $\|x(0)\|_{m}=\delta$. Then, for $k=-m,-m+$ $1, \ldots, 0$,

$$
\begin{aligned}
V(x(k), k) & \leq \gamma^{k} \lambda_{\max }(H)|x(k)|^{2} \\
& \leq \gamma^{k} \lambda_{\max }(H)\|x(0)\|_{m}^{2} \leq \gamma^{k} \lambda_{\max }(H) \delta^{2} \\
& =\gamma^{k} \lambda_{\max }(H) \frac{\varepsilon^{2}}{\varphi(H)}=\gamma^{k} \varepsilon^{2} \lambda_{\min }(H) \\
& \leq \varepsilon^{2} \lambda_{\min }(H),
\end{aligned}
$$

i.e.,

$$
V(x(k), k) \leq \varepsilon^{2} \lambda_{\min }(H) .
$$

Below, we prove that (10) is valid for $k=1,2, \ldots$, too. Assume, on the contrary, that (10) is not always valid. Then, an integer $k^{*}>0$ exists such that, for $k=-m,-m+1, \ldots, k^{*},(10)$ holds, and, for $k=k^{*}+1$,

$$
V\left(x\left(k^{*}+1, k^{*}+1\right)\right)>\varepsilon^{2} \lambda_{\min }(H) .
$$

Inequality (11) implies that, for $k=-m,-m+1, \ldots, k^{*}$,

$$
\begin{aligned}
\gamma^{k} \lambda_{\min }(H)|x(k)|^{2} & \leq V(x(k), k) \leq \varepsilon^{2} \lambda_{\min }(H) \\
& <V\left(x\left(k^{*}+1\right), k^{*}+1\right) \\
& \leq \gamma^{k^{*}+1} \lambda_{\max }(H)\left|x\left(k^{*}+1\right)\right|^{2}
\end{aligned}
$$

and

$$
\begin{aligned}
|x(k)|<\gamma^{\left(k^{*}+1-k\right) / 2} \sqrt{\varphi(H)}\left|x\left(k^{*}+1\right)\right|, & \\
k & =-m,-m+1, \ldots, k^{*} .
\end{aligned}
$$

Now compute

$$
\begin{aligned}
& \Delta V\left(x\left(k^{*}\right), k^{*}\right)=V\left(x\left(k^{*}+1\right), k^{*}+1\right) \\
& -V\left(x\left(k^{*}\right), k^{*}\right)=\gamma^{k^{*}+1} x^{T}\left(k^{*}+1\right) H x\left(k^{*}+1\right) \\
& -\gamma^{k^{*}} x^{T}\left(k^{*}\right) H x\left(k^{*}\right) \\
& =\gamma^{k^{*}+1}\left[A x\left(k^{*}\right)+\sum_{i=1}^{s} B_{i} x\left(k^{*}-m_{i}\right)\right]^{T} \\
& \cdot H\left[A x\left(k^{*}\right)+\sum_{i=1}^{s} B_{i} x\left(k^{*}-m_{i}\right)\right]-\gamma^{k^{*}} x^{T}\left(k^{*}\right) \\
& \cdot H x\left(k^{*}\right) .
\end{aligned}
$$

Rearranging this computation, we derive

$$
\begin{aligned}
\Delta V( & \left.x\left(k^{*}\right), k^{*}\right) \\
= & -\gamma^{k^{*}+1} x^{T}\left(k^{*}\right)\left[H-A^{T} H A\right] x\left(k^{*}\right) \\
& +2 \gamma^{k^{*}+1} x^{T}\left(k^{*}\right) A^{T} H \sum_{i=1}^{s} B_{i} x\left(k^{*}-m_{i}\right) \\
& +\gamma^{k^{*}+1} \sum_{i, j=1}^{s} x^{T}\left(k^{*}-m_{i}\right) B_{i}^{T} H B_{j} x\left(k^{*}-m_{j}\right) \\
& +\gamma^{k^{*}}(\gamma-1) x^{T}\left(k^{*}\right) H x\left(k^{*}\right) .
\end{aligned}
$$


We estimate the first difference and use the assumption that the matrix $H$ is a solution of equation (5); therefore,

$$
\begin{aligned}
\Delta V(x & \left.\left(k^{*}\right), k^{*}\right) \\
\leq & -\gamma^{k^{*}+1} \lambda_{\min }(C)\left|x\left(k^{*}\right)\right|^{2} \\
& +2 \gamma^{k^{*}+1} \sum_{i=1}^{s}\left|A^{T} H B_{i}\right|\left|x\left(k^{*}\right)\right|\left|x\left(k^{*}-m_{i}\right)\right| \\
& +\gamma^{k^{*}+1} \sum_{i, j=1}^{s}\left|B_{i}^{T} H B_{j}\right|\left|x\left(k^{*}-m_{i}\right)\right|\left|x\left(k^{*}-m_{j}\right)\right| \\
& +\gamma^{k^{*}}(\gamma-1) \lambda_{\max }(H)\left|x\left(k^{*}\right)\right|^{2}
\end{aligned}
$$

and

$$
\begin{aligned}
& \Delta V\left(x\left(k^{*}\right), k^{*}\right) \leq-\gamma^{k^{*}+1} \lambda_{\min }(C)\left|x\left(k^{*}\right)\right|^{2} \\
& +\gamma^{k^{*}+1} \sum_{i=1}^{s}\left|A^{T} H B_{i}\right|\left[\left|x\left(k^{*}\right)\right|^{2}+\left|x\left(k^{*}-m_{i}\right)\right|^{2}\right] \\
& +\frac{1}{2} \gamma^{k^{*}+1} \sum_{i, j=1}^{s}\left|B_{i}^{T} H B_{j}\right| \\
& \cdot\left[\left|x\left(k^{*}-m_{i}\right)\right|^{2}+\left|x\left(k^{*}-m_{j}\right)\right|^{2}\right]+\gamma^{k^{*}}(\gamma-1) \\
& \cdot \lambda_{\max }(H)\left|x\left(k^{*}\right)\right|^{2} .
\end{aligned}
$$

Now we apply inequality (13) to get

$$
\begin{aligned}
& \Delta V\left(x\left(k^{*}\right), k^{*}\right) \leq-\gamma^{k^{*}+1} \lambda_{\min }(C)\left|x\left(k^{*}\right)\right|^{2} \\
& +\gamma^{k^{*}+1} \sum_{i=1}^{s}\left|A^{T} H B_{i}\right| \\
& \cdot\left[\left|x\left(k^{*}\right)\right|^{2}+\gamma^{m_{i}+1} \varphi(H)\left|x\left(k^{*}+1\right)\right|^{2}\right]+\frac{1}{2} \\
& \cdot \gamma^{k^{*}+1} \sum_{i, j=1}^{s}\left|B_{i}^{T} H B_{j}\right|\left[\gamma^{m_{i}+1}+\gamma^{m_{j}+1}\right] \varphi(H) \\
& \cdot\left|x\left(k^{*}+1\right)\right|^{2}+\gamma^{k^{*}}(\gamma-1) \lambda_{\max }(H)\left|x\left(k^{*}\right)\right|^{2}
\end{aligned}
$$

and

$$
\begin{gathered}
\Delta V\left(x\left(k^{*}\right), k^{*}\right) \leq-\gamma^{k^{*}+1}\left[\lambda_{\min }(C)-\sum_{i=1}^{s}\left|A^{T} H B_{i}\right|\right. \\
\left.-\frac{\gamma-1}{\gamma} \lambda_{\max }(H)\right]\left|x\left(k^{*}\right)\right|^{2}+\frac{1}{2} \gamma^{\left(k^{*}+2\right)} \varphi(H) \\
.\left[2 \sum_{i=1}^{s} \gamma^{m_{i}}\left|A^{T} H B_{i}\right|\right. \\
\left.+\sum_{i, j=1}^{s}\left[\gamma^{m_{i}+1}+\gamma^{m_{j}+1}\right]\left|B_{i}^{T} H B_{j}\right|\right]\left|x\left(k^{*}+1\right)\right|^{2} .
\end{gathered}
$$

Inequality

$$
\lambda_{\text {min }}(C)-\sum_{i=1}^{s}\left|A^{T} H B_{i}\right|-\frac{\gamma-1}{\gamma} \lambda_{\max }(H)>0
$$

can be deduced from the assumption $L_{3} \geq 0$. Therefore, utilizing (8),

$$
\begin{aligned}
& \Delta V\left(x\left(k^{*}\right), k^{*}\right) \leq-\gamma\left[\lambda_{\min }(C)-\sum_{i=1}^{s}\left|A^{T} H B_{i}\right|\right. \\
& \left.-\frac{\gamma-1}{\gamma} \lambda_{\max }(H)\right] \frac{V\left(x\left(k^{*}\right), k^{*}\right)}{\lambda_{\max }(H)}+\frac{1}{2} \gamma \varphi(H) \\
& \cdot\left[2 \sum_{i=1}^{s} \gamma^{m_{i}}\left|A^{T} H B_{i}\right|\right. \\
& \left.+\sum_{i, j=1}^{s}\left[\gamma^{m_{i}+1}+\gamma^{m_{j}+1}\right]\left|B_{i}^{T} H B_{j}\right|\right] \\
& +\frac{V\left(x\left(k^{*}+1\right), k^{*}+1\right)}{\lambda_{\min }(H)} .
\end{aligned}
$$

Since $\Delta V\left(x\left(k^{*}\right), k^{*}\right)=V\left(x\left(k^{*}+1\right), k^{*}+1\right)-V\left(x\left(k^{*}\right), k^{*}\right)$, we get

$$
\begin{aligned}
& {\left[1-\frac{1}{2} \gamma \frac{\varphi(H)}{\lambda_{\min }(H)}\left[2 \sum_{i=1}^{s} \gamma^{m_{i}}\left|A^{T} H B_{i}\right|\right.\right.} \\
& \left.+\sum_{i, j=1}^{s}\left[\gamma^{m_{i}+1}+\gamma^{m_{j}+1}\right]\left|B_{i}^{T} H B_{j}\right|\right] V\left(x\left(k^{*}+1\right),\right. \\
& \left.k^{*}+1\right) \leq\left[1-\frac{\gamma}{\lambda_{\max }(H)}\left[\lambda_{\min }(C)-\sum_{i=1}^{s}\left|A^{T} H B_{i}\right|\right.\right. \\
& \left.\left.-\frac{\gamma-1}{\gamma} \lambda_{\max }(H)\right]\right] V\left(x\left(k^{*}\right), k^{*}\right) .
\end{aligned}
$$

This inequality can be rewritten as

$$
\begin{gathered}
\frac{L_{2}}{\lambda_{\min }(H)} V\left(x\left(k^{*}+1\right), k^{*}+1\right) \\
\leq \frac{L_{1}}{\lambda_{\max }(H)} V\left(x\left(k^{*}\right), k^{*}\right)
\end{gathered}
$$

or as

$$
V\left(x\left(k^{*}+1\right), k^{*}+1\right) \leq \Theta \cdot V\left(x\left(k^{*}\right), k^{*}\right)
$$

where

$$
\Theta:=\frac{\mathrm{L}_{1}}{L_{2} \varphi(H)}>0 .
$$

Now we prove that

$$
\Theta \leq 1
$$


Inequality (26) is equivalent with an inequality

$$
\begin{aligned}
& \lambda_{\max }(H)-\gamma\left[\lambda_{\min }(C)-\sum_{i=1}^{s}\left|A^{T} H B_{i}\right|-\frac{\gamma-1}{\gamma}\right. \\
& \left.\cdot \lambda_{\max }(H)\right] \leq\left[\lambda_{\min }(H)-\frac{1}{2} \gamma \varphi(H)\right. \\
& \cdot\left[2 \sum_{i=1}^{s} \gamma^{m_{i}}\left|A^{T} H B_{i}\right|\right. \\
& \left.\left.+\sum_{i, j=1}^{s}\left[\gamma^{m_{i}+1}+\gamma^{m_{j}+1}\right]\left|B_{i}^{T} H B_{j}\right|\right]\right] \frac{\lambda_{\max }(H)}{\lambda_{\min }(H)} .
\end{aligned}
$$

After some simplification, we get

$$
\begin{gathered}
\lambda_{\min }(C)-\sum_{i=1}^{s}\left|A^{T} H B_{i}\right|-\frac{\gamma-1}{\gamma} \lambda_{\max }(H) \geq \frac{1}{2} \varphi^{2}(H) \\
\cdot\left[2 \sum_{i=1}^{s} \gamma^{m_{i}}\left|A^{T} H B_{i}\right|\right. \\
\left.+\sum_{i, j=1}^{s}\left[\gamma^{m_{i}+1}+\gamma^{m_{j}+1}\right]\left|B_{i}^{T} H B_{j}\right|\right],
\end{gathered}
$$

which is equivalent with the inequality $L_{3} \geq 0$. Then (24), (26), and (10) imply

$$
\begin{aligned}
& V\left(x\left(k^{*}+1\right), k^{*}+1\right) \leq \Theta \cdot V\left(x\left(k^{*}\right), k^{*}\right) \\
& \leq V\left(x\left(k^{*}\right), k^{*}\right) \leq \varepsilon^{2} \lambda_{\min }(H) .
\end{aligned}
$$

This inequality contradicts (11). Then, inequality (11) is impossible and (10) holds for every $k=1,2, \ldots$. Moreover, (8) and (10) imply

$$
\begin{aligned}
\gamma^{k} \lambda_{\min }(H)|x(k)|^{2} & \leq V(x(k), k) \leq \varepsilon^{2} \lambda_{\min }(H) \\
& =\delta^{2} \lambda_{\max }(H) \\
& =\|x(0)\|_{m}^{2} \lambda_{\max }(H),
\end{aligned}
$$

i.e., the inequality

$$
\gamma^{k} \lambda_{\min }(H)|x(k)|^{2} \leq\|x(0)\|_{m}^{2} \lambda_{\max }(H), \quad k \geq 1,
$$

equivalent with (7).

\section{Concluding Remarks}

Based on the investigations on exponential stability published previously, the present paper brings in Theorem 1 new results. The exponential rate of convergence of solutions is studied in [1] assuming that $\operatorname{det} A \neq 0$; therefore, the results are independent. Let us discuss the independence of the results of other sources listed in the references. The criteria for the exponential stability of nonlinear difference systems, for example, are proved in $[11,14]$. The nonlinearities are estimated by some linear terms with matrices having nonnegative entries with the sums of such matrices being, for example, a constant nonnegative matrix with a spectrum less than 1. In general, an attempt to estimate the right-hand sides of the systems by a nonnegative matrix does not provide a matrix with a spectrum less than 1 and the results are independent. For special classes of equations, sharp criteria (depending on delay) for detecting asymptotic stability are proved in $[2,3]$. The following example illustrates the abovementioned independency of results.

Example 2. Let $n=s=2$ and let system (1) be of the form

$$
\begin{aligned}
& x_{1}(k+1)=x_{1}(k)+x_{2}(k)+\mu x_{2}\left(k-m_{1}\right), \\
& x_{2}(k+1)=-x_{1}(k)-x_{2}(k)+v x_{1}\left(k-m_{2}\right)
\end{aligned}
$$

where $k \geq 0$ and $\mu$ and $\nu$ are constants. We show that Theorem 1 is applicable if $|\mu|$ and $|\nu|$ are sufficiently small. We have

$$
\begin{aligned}
A & =\left(\begin{array}{cc}
1 & 1 \\
-1 & -1
\end{array}\right), \\
B_{1} & =\left(\begin{array}{ll}
0 & \mu \\
0 & 0
\end{array}\right), \\
B_{2} & =\left(\begin{array}{ll}
0 & 0 \\
\nu & 0
\end{array}\right) .
\end{aligned}
$$

Lyapunov equation (5) is satisfied, e.g., for

$$
\begin{aligned}
& C=\left(\begin{array}{cc}
0.9 & 0.9 \\
0.9 & 1
\end{array}\right), \\
& H=\left(\begin{array}{cc}
1 & 1 \\
1 & 1.1
\end{array}\right) .
\end{aligned}
$$

Then, $\lambda_{\text {max }}(H) \doteq 2.0512492, \lambda_{\text {min }}(H) \doteq 0.0487508$, $\lambda_{\text {min }}(C) \doteq 0.0486122$, and $\varphi(H) \doteq$ 42.0762336. Simple computations result in

$$
\begin{aligned}
& \left|A^{T} H B_{1}\right|=0, \\
& \left|A^{T} H B_{2}\right|=0.1 \sqrt{2} \nu, \\
& \left|B_{1}^{T} H B_{1}\right|=\mu^{2}, \\
& \left|B_{2}^{T} H B_{2}\right|=1.1 \nu^{2}, \\
& \left|B_{1}^{T} H B_{2}\right|=\mu \nu,
\end{aligned}
$$




$$
\begin{aligned}
L_{1} & =\gamma\left[\lambda_{\max }(H)-\lambda_{\min }(C)+\sum_{j=1}^{2}\left|A^{T} H B_{j}\right|\right] \\
& \doteq \gamma[2.0026370+0.1 \sqrt{2} \nu] \\
L_{2} & =\lambda_{\min }(H)-\frac{1}{2} \gamma \varphi(H)\left[2 \sum_{i=1}^{2} \gamma^{m_{i}}\left|A^{T} H B_{i}\right|\right. \\
& \left.+\sum_{i, j=1}^{2}\left[\gamma^{m_{i}+1}+\gamma^{m_{j}+1}\right]\left|B_{i}^{T} H B_{j}\right|\right] \doteq 0.0487508 \\
& -\gamma 42.0762336\left[\gamma^{m_{2}} 0.1 \sqrt{2} \nu+\gamma^{m_{1}+1} \mu^{2}\right. \\
& \left.+\left(\gamma^{m_{1}+1}+\gamma^{m_{2}+1}\right) \mu \nu+\gamma^{m_{2}+1} 1.1 \nu^{2}\right]
\end{aligned}
$$

and

$$
\begin{aligned}
L_{3} & =\lambda_{\min }(C)-\sum_{i=1}^{2}\left|A^{T} H B_{i}\right|-\frac{\gamma-1}{\gamma} \lambda_{\max }(H)-\frac{1}{2} \\
& \cdot \varphi^{2}(H)\left[2 \sum_{i=1}^{2} \gamma^{m_{i}}\left|A^{T} H B_{i}\right|\right. \\
& \left.+\sum_{i, j=1}^{2}\left[\gamma^{m_{i}+1}+\gamma^{m_{j}+1}\right]\left|B_{i}^{T} H B_{j}\right|\right] \doteq 0.0486122 \\
& -0.1 \sqrt{2} \nu-\frac{\gamma-1}{\gamma} 2.0512492-(42.0762336)^{2} \\
& \cdot\left[\gamma^{m_{2}} 0.1 \sqrt{2} \nu+\gamma^{m_{1}+1} \mu^{2}+\left(\gamma^{m_{1}+1}+\gamma^{m_{2}+1}\right) \mu \nu\right. \\
& \left.+\gamma^{m_{2}+1} 1.1 \nu^{2}\right] .
\end{aligned}
$$

Theorem 1 is applicable if $|\mu|$ and $|\nu|$ are sufficiently small since this implies $L_{i}>0, i=1,2$, and, if the expression

$$
\begin{array}{r}
0.0486122-\frac{\gamma-1}{\gamma} 2.0512492 \\
=\frac{2.0512492}{\gamma}-2.0026370
\end{array}
$$

is positive, provided that $\gamma>1$; that is, if

$$
1<\gamma<\frac{2.0512492}{2.0026370} \doteq 1.0242741
$$

then $L_{3}>0$ as well. In such a case, for an arbitrary solution $x(k)=\left(x_{1}(k), x_{2}(k)\right)^{T}$ of system (32), (33), the estimate

$$
\begin{aligned}
|x(k)| & \leq \sqrt{\varphi(H)}\|x(0)\|_{m} \gamma^{-k / 2} \\
& \doteq 42.0762336\|x(0)\|_{m} \gamma^{-k / 2}, \quad k \geq 1
\end{aligned}
$$

holds.
Since $\operatorname{det} A=0$ in the above example, the results of the paper [1] are not applicable to system (32), (33). Moreover, an attempt to apply results of $[11,14]$ is not successful since the sum of matrices $A^{*}, B_{1}^{*}$, and $B_{2}^{*}$, defined by replacing the entries in the previously given matrices $A, B_{1}$, and $B_{2}$ by their absolute values, leads to a matrix

$$
\begin{aligned}
U & :=A^{*}+B_{1}^{*}+B_{2}^{*}=\left(\begin{array}{ll}
1 & 1 \\
1 & 1
\end{array}\right)+\left(\begin{array}{cc}
0 & |\mu| \\
0 & 0
\end{array}\right)+\left(\begin{array}{cc}
0 & 0 \\
|\nu| & 0
\end{array}\right) \\
& =\left(\begin{array}{cc}
1 & 1+|\mu| \\
1+|\nu| & 1
\end{array}\right)
\end{aligned}
$$

whose eigenvalues are $\lambda_{1,2}(U)=1 \pm \sqrt{(1+|\mu|)(1+|\nu|)}$, and, obviously, $\rho(U) \geq 1$.

Finally, we compare the results published in [4-7] with Theorem 1 . The assumptions of Theorem 1 are, for the reduced case $s=1$ of a single delay, weaker than those of Theorem 2 in [7]. In [4] an analysis of Theorem 2 is carried out. Although the results are independent, a limiting process (for $\gamma \longrightarrow 1^{+}$) indicates that the conditions of the main result in [7] are, in general, more restrictive. Now we will demonstrate that, with respect to the derived estimates of the norms of solutions, the situation is just the opposite and that the estimation (7) is, in general, better than that in $[4$, Theorem 2$]$. The last estimation mentioned says that (below, $s, A, B_{i}, i=1 \ldots, s, H$ and $C$ are the same as in the paper)

$$
|x(k)| \leq \sqrt{\varphi(H)}\|x(0)\|_{m} \Theta^{k / 2(m+1)}(H), \quad k \geq 1,
$$

where

$$
\begin{aligned}
& \Theta(H) \\
& :=\frac{1}{\lambda_{\max }(H)}\left[L(H)-\sum_{i=1}^{s} L_{i}(H)+s \lambda_{\min }(H)\right], \\
& L(H):=\lambda_{\max }(H)-\lambda_{\min }(C)+\sum_{j=1}^{s}\left|A^{T} H B_{j}\right|, \\
& L_{i}(H) \\
& \quad:=\lambda_{\min }(H)-\varphi(H)\left[\left|A^{T} H B_{i}\right|+\sum_{j=1}^{s}\left|B_{i}^{T} H B_{j}\right|\right],
\end{aligned}
$$

if $\rho(A)<1, C$ is a fixed positive definite matrix, matrix $H$ solves the corresponding Lyapunov matrix equation (5), and

$$
L(H)-\sum_{i=1}^{s} L_{i}(H)<\lambda_{\max }(H)-s \lambda_{\min }(H),
$$

$$
L(H)>0 \text {. }
$$

Assuming that $\left|B_{i}\right| \longrightarrow 0, i=1, \ldots, n$, we deduce that for (44) to hold, the following is necessary:

$$
\lambda_{\max }(H)-\lambda_{\min }(C)>0,
$$


the limiting value of $\Theta(H)$ is

$$
\Theta(H) \doteq \frac{\lambda_{\text {max }}(H)-\lambda_{\text {min }}(C)}{\lambda_{\text {max }}(H)},
$$

and (42) can approximately be written as

$$
\begin{aligned}
& |x(k)| \\
& \leq \sqrt{\varphi(H)}\|x(0)\|_{m}\left[\frac{\lambda_{\max }(H)-\lambda_{\min }(C)}{\lambda_{\max }(H)}\right]^{k /(2(m+1))}, \\
& k \geq 1 .
\end{aligned}
$$

Considering the same limiting process as above, for the validity of (7), an analysis of $L_{i}, i=1,2,3$ implies that inequality (45) must hold in addition to inequality

$$
\lambda_{\min }(C)-\lambda_{\max }(H)+\frac{1}{\gamma} \lambda_{\max }(H)>0
$$

derived from the assumption $L_{3} \geq 0$. Inequality (48), together with the assumption $\gamma>1$, yields

$$
1<\gamma<\frac{\lambda_{\max }(H)}{\lambda_{\max }(H)-\lambda_{\min }(C)}
$$

and (7) can be approximatively written as

$$
\begin{aligned}
& |x(k)| \\
& \quad \leq \sqrt{\varphi(H)}\|x(0)\|_{m}\left[\frac{\lambda_{\max }(H)-\lambda_{\min }(C)}{\lambda_{\max }(H)}\right]^{k / 2}, \\
& k \geq 1 .
\end{aligned}
$$

Obviously, estimation (50) is (due to the absence of the maximal delay $m$ ) better than estimation (47). We finish this part with a remark that the results of [5] are generalized in [4]. Results of [6] are on the exponential stability of linear perturbed systems with a single delay. Among others, it is proved [6, Theorem 3] that inequality (50) holds for nondelayed linear systems

$$
x(k+1)=A x(k), \quad k=0,1, \ldots
$$

\section{Data Availability}

No data were used to support this study.

\section{Conflicts of Interest}

The authors declare that there are no conflicts of interest regarding the publication of this paper.

\section{Acknowledgments}

The first, third, and fourth authors have been supported by the Czech Science Foundation under Project 16-08549S. Their work has been realized in CEITEC-Central European
Institute of Technology with research infrastructure supported by Project CZ.1.05/1.1.00/02.0068 financed from European Regional Development Fund. The second author has been supported by the Grant FEKT-S-17-4225 of Faculty of Electrical Engineering and Communication, Brno University of Technology. An earlier presentation of preliminary results was introduced on Thursday (May 19, 2016) at Faculty of Physics and Mathematics, University of Latvia.

\section{References}

[1] A. S. Bychkov and D. Y. Khusainov, "Exponential convergence estimates for delay difference systems," Journal of Differential Equations, vol. 38, no. 9, pp. 1368-1370, 2002, translation from Differ. Uravn. vol. 38, no. 9, pp. 1285-1287, 2002.

[2] J. Čermák, "Stability conditions for linear delay difference equations: a survey and perspective," Tatra Mountains Mathematical Publications, vol. 63, pp. 1-29, 2015.

[3] J. Čermák and J. Jánský, "Stability switches in linear delay difference equations," Applied Mathematics and Computation, vol. 243, pp. 755-766, 2014.

[4] J. Diblík, "Exponential stability of linear discrete systems with variable delays via Lyapunov second method," Discrete Dynamics in Nature and Society, vol. 2017, Article ID 7417909, 9 pages, 2017.

[5] J. Diblík, D. Y. Khusainov, J. Baštinec, and A. S. Sirenko, "Exponential stability of linear discrete systems with constant coefficients and single delay," Applied Mathematics Letters, vol. 51, pp. 68-73, 2016.

[6] J. Diblík, D. Y. Khusainov, J. Baštinec, and A. S. Sirenko, "Exponential stability of perturbed linear discrete systems," Advances in Difference Equations, vol. 2016, pp. 1-20, 2016.

[7] J. Diblík, D. Y. Khusainov, J. Baštinec, and A. S. Sirenko, "Stability and exponential stability of linear discrete systems with constant coefficients and single delay," Applied Mathematics and Computation, vol. 269, Article ID 21439, pp. 9-16, 2015.

[8] E. Kaslik, "Stability results for a class of difference systems with delay," Advances in Difference Equations, vol. 2009, Article ID 938492, 13 pages, 2009.

[9] M. M. Kipnis and R. M. Nigmatulin, "Stability of trinomial linear difference equations with two delays," Automation and Remote Control, no. 11, pp. 1710-1723, 2004.

[10] S. A. Kuruklis, "The asymptotic stability of $x_{n+1}-a x_{n}+b x_{n-k}=$ 0," Journal of Mathematical Analysis and Applications, vol. 188, no. 3, pp. 719-731, 1994.

[11] E. Liz, "Stability of non-autonomous difference equations: simple ideas leading to useful results," Journal of Difference Equations and Applications, vol. 17, no. 2, pp. 203-220, 2011.

[12] E. Liz and J. B. Ferreiro, "A note on the global stability of generalized difference equations," Applied Mathematics Letters, vol. 15 , no. 6, pp. 655-659, 2002.

[13] M. Medved' and L. Škripková, "Sufficient conditions for the exponential stability of delay difference equations with linear parts defined by permutable matrices," Electronic Journal of Qualitative Theory of Differential Equations, vol. 22, pp. 1-13, 2012.

[14] P. H. Ngoc and L. T. Hieu, "New criteria for exponential stability of nonlinear difference systems with time-varying delay," International Journal of Control, vol. 86, no. 9, pp. 16461651, 2013. 
[15] R. P. Agarwal, Difference Equations and Inequalities. Theory, Methods and Applications, Monographs and Textbooks in Pure and Applied Mathematics, Marcel Dekker, Inc., New York, NY, USA, 2nd edition, 2000.

[16] S. Elaydi, "An introduction to difference equations," in Undergraduate Texts in Mathematics, Springer, 3rd edition, 2005.

[17] W. B. Jones and W. J. Thron, Continued Fractions, Analytic Theory and Applications, vol. 11 of Encyclopedia of Mathematics and its Applications, Addison-Wesley Publishing Co., Reading, MA, USA, 1980. 


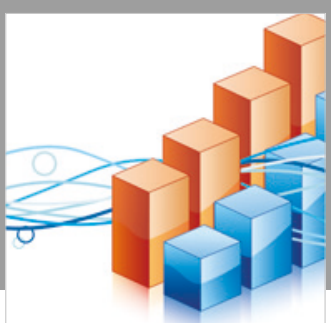

Advances in

Operations Research

\section{-n-m}
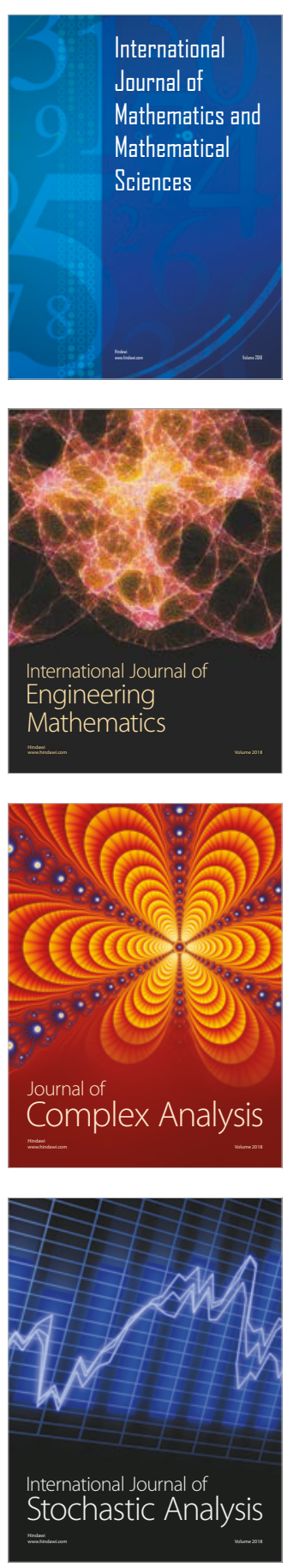
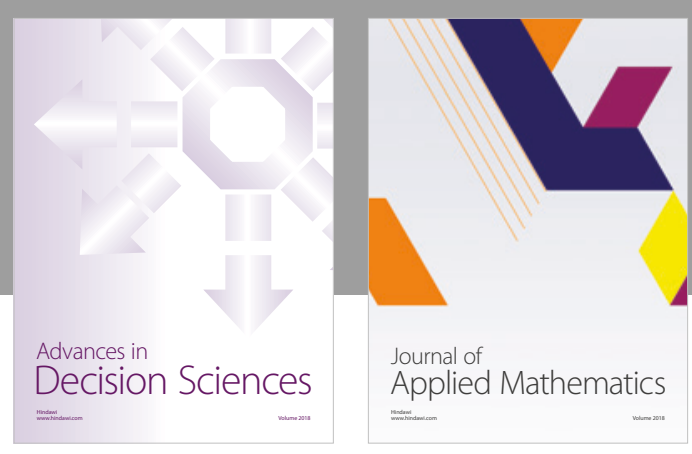

Journal of

Applied Mathematics
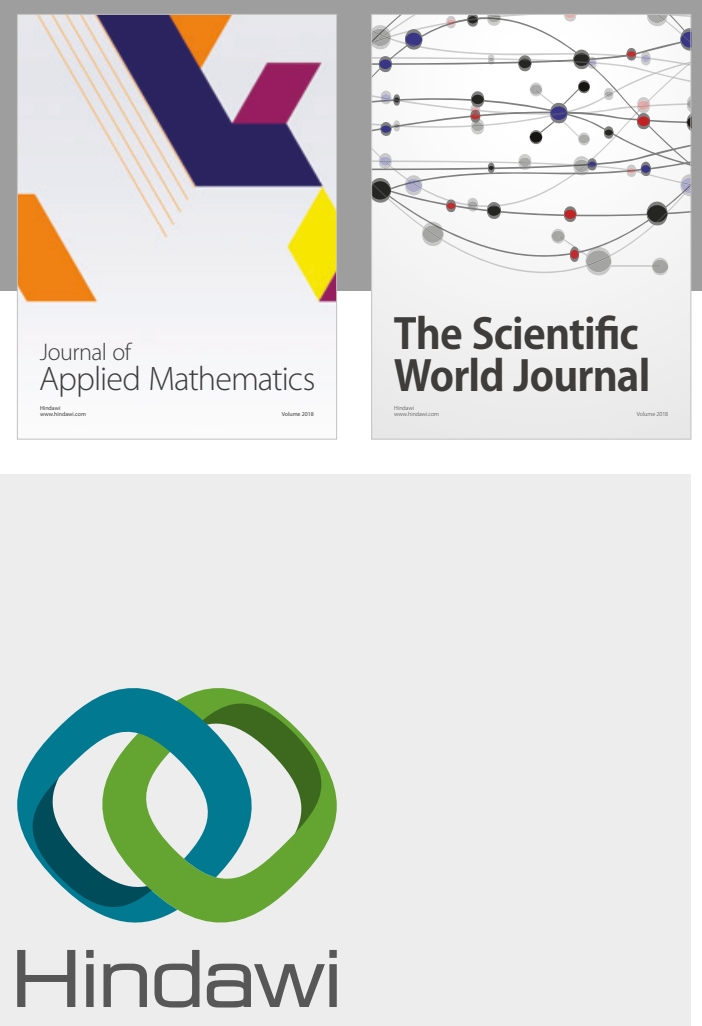

Submit your manuscripts at

www.hindawi.com

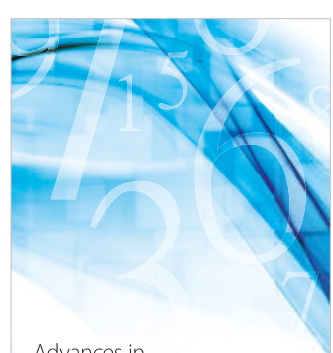

Advances in
Numerical Analysis
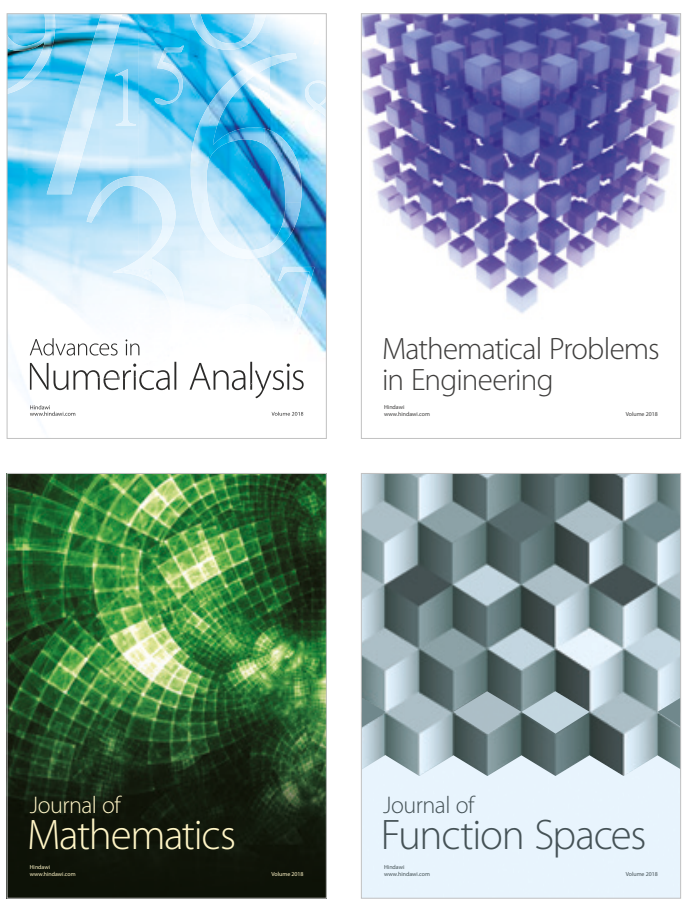

Mathematical Problems in Engineering

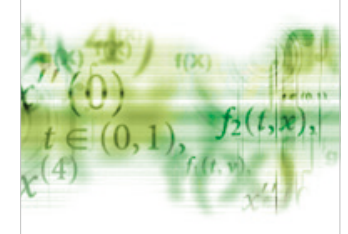

International Journal of

Differential Equations

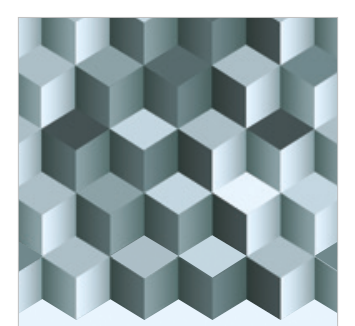

Journal of

Function Spaces

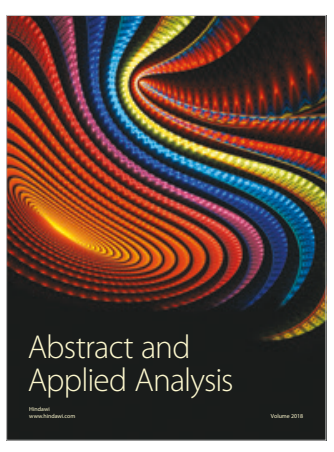

The Scientific

World Journal

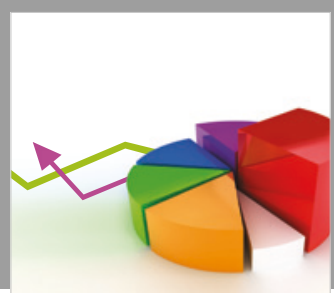

Journal of

Probability and Statistics
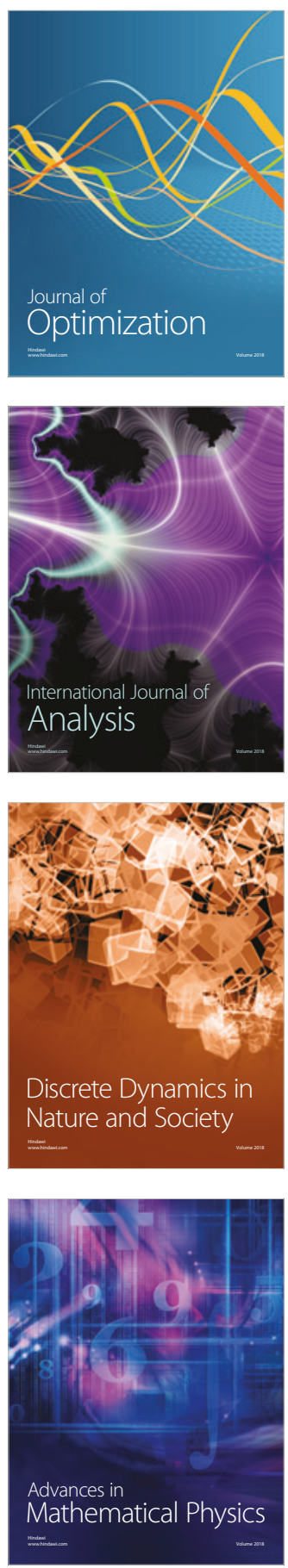